\title{
Efficient BER Simulation of Orthogonal Space-Time Block Codes in Nakagami- $m$ Fading
}

\author{
Lennert Jacobs and Marc Moeneclaey \\ Ghent University, TELIN Department, DIGCOM Group \\ Sint-Pietersnieuwstraat 41, B-9000 Gent, Belgium \\ \{Lennert.Jacobs,Marc.Moeneclaey\}@ telin.ugent.be
}

\begin{abstract}
In this contribution, we present a simple but efficient importance sampling technique to speed up Monte Carlo simulations for bit error rate estimation of orthogonal space-time block codes on spatially correlated Nakagami- $m$ fading channels. While maintaining the actual distributions for the channel noise and the data symbols, we derive a convenient biased distribution for the fading channel that is shown to result in impressive efficiency gains up to multiple orders of magnitude.
\end{abstract}

\section{INTRODUCTION}

The bit error rate (BER) is a fundamental measure to evaluate the performance of digital communication systems. When analytical BER calculation is not feasible due to complexity, Monte-Carlo (MC) simulations can be applied to estimate the BER. However, the computation time associated with these simulations may be prohibitively long, especially when low BERs are targeted and many bits must be sent to generate a sufficient number of bit errors. A substantial reduction of the simulation time can be achieved by employing the wellknown technique of importance sampling (IS) [1], which aims to reduce the variance of the BER estimator by using a biased distribution for the input variables that increases the bit error probability during simulation. By weighting the results with the ratios of the actual to the biased probability density functions, an unbiased BER estimate with lower variance can be obtained.

Although the application of IS is well documented, the search for a convenient biased distribution remains a major issue in many papers, e.g., [2]-[5]. In order to speed up the simulation of orthogonal space-time block codes (OSTBCs) on i.i.d. Rayleigh-faded multiple-input multiple-output (MIMO) channels, Nguyen et al. show in [6] that scaling the channel distribution is more efficient than scaling the noise distribution. However, an optimal biased channel distribution was not derived. In line with the conclusions of Nguyen et al., we propose in this work an IS technique for the simulation of OSTBCs where we keep the distributions for the channel noise and the data symbols unchanged and use a biased channel distribution. However, we assume a general MIMO Nakagami- $m$ fading channel, which includes Rayleigh fading as a special case, and allow spatial correlation between the different channel coefficients. Moreover, we do propose a convenient importance sampling distribution. To the best of our knowledge, such practical biased distribution for simulation over non-Rayleigh fading channels is not yet available from the literature. Al- though perfect channel knowledge (PCK) is assumed for the derivation of the biased distribution, it allows accurate BER estimation in a wide range of scenarios, such as pilot-based [7] or blind channel estimation, and in the presence of residual frequency offset, IQ imbalance, and phase noise. In addition, the technique also applies to single-input multiple-output (SIMO) systems with maximal-ratio combining (MRC) and can easily be extended to the case of multicarrier communication on dispersive MIMO channels, using for instance OSTBCs or orthogonal space-frequency block codes. Numerical results illustrate that impressive efficiency gains up to multiple orders of magnitude can be obtained using the proposed IS technique.

\section{SYSTEM MODEL}

We assume a MIMO communication system using $L_{\mathrm{t}}$ transmit antennas and $L_{\mathrm{r}}$ receive antennas. The $L=L_{\mathrm{t}} L_{\mathrm{r}}$ complex-valued coefficients describing the frequency-flat fading channels between the transmit and receive antennas are comprised in a $L_{\mathrm{r}} \times L_{\mathrm{t}}$ channel matrix $\mathbf{H}$. In the case of orthogonal space-time block coding [8], a symbol vector $\mathbf{s}$ consisting of $N_{\mathrm{s}}$ information symbols $s_{i}$, with $i=1, \ldots, N_{\mathrm{s}}$, is transformed into a $L_{\mathrm{t}} \times K_{\mathrm{c}}$ code matrix $\mathbf{C}(\mathbf{s})$, which is transmitted over the MIMO channel. The code matrix $\mathbf{C}(\mathbf{s})$ satisfies the orthogonality condition

$$
\mathbf{C}(\mathbf{s}) \mathbf{C}^{H}(\mathbf{s})=\lambda\|\mathbf{s}\|^{2} \mathbf{I}_{L_{\mathrm{t}}},
$$

with scaling factor $\lambda \triangleq K_{\mathrm{c}} / N_{\mathrm{s}}$, such that $E_{\mathrm{s}} \triangleq \mathbb{E}\left[\left|s_{i}\right|^{2}\right]$ denotes the average transmitted energy per symbol interval per antenna. The corresponding received $L_{\mathrm{r}} \times K_{\mathrm{c}}$ matrix $\mathbf{R}$ is given by

$$
\mathbf{R}=\mathbf{H C}(\mathbf{s})+\mathbf{W},
$$

where the AWGN matrix $\mathbf{W}$ consists of independent and identically distributed (i.i.d.) zero mean circularly symmetric complex Gaussian (ZMCSCG) random variables (RVs).

\section{IMPORTANCE SAMPLING}

Defining $\mathbf{h}=\operatorname{vec}(\mathbf{H})$ and $\mathbf{w}=\operatorname{vec}(\mathbf{W})$, and collecting the input vectors $\mathbf{s}, \mathbf{w}$, and $\mathbf{h}$ into a single vector $\mathbf{x}=[\mathbf{s}, \mathbf{w}, \mathbf{h}]$, the average BER can be written as

$$
P_{\mathrm{b}}=\mathbb{E}[F(\mathbf{x})]
$$

where $F(\mathbf{x})$ is the fraction of bit errors corresponding to given $\mathbf{x}$, and $\mathbb{E}[$.$] denotes expectation over the PDF p(\mathbf{x})$ of the 
vector $\mathrm{x}$. With $N_{\mathrm{b}}$ denoting the number of bits contained in the symbol vector $\mathbf{s}$, the fraction $F(\mathbf{x})$ is given by

$$
F(\mathbf{x})=\frac{1}{N_{\mathrm{b}}} \sum_{n=1}^{N_{\mathrm{b}}} I_{n}(\mathbf{x}),
$$

where $I_{n}(\mathbf{x})$ equals 1 when the decision about the $n$th bit is wrong, and zero otherwise.

When analytical averaging over $\mathrm{x}$ is too complex, a closedform BER expression cannot be obtained and MC simulations are required to estimate the BER. In particular, by independently generating a set of $N$ realizations $\left\{\mathbf{x}_{i}\right\}$ of the input vector $\mathbf{x}$ according to the PDF $p(\mathbf{x})$, and simulating for each realization the system operations that yield the bit decisions at the receiver, the average BER is estimated as the ratio of the number of counted bit errors to the total number of bits transmitted

$$
\hat{P}_{\mathrm{b}} \triangleq \frac{1}{N} \sum_{i=1}^{N} F\left(\mathbf{x}_{i}\right) .
$$

Taking into account that $\mathbb{E}\left[\hat{P}_{\mathrm{b}}\right]=P_{\mathrm{b}}$ and that the vectors $\mathbf{x}_{i}$ in (5) are independently generated, the variance of the BER estimator $\hat{P}_{\mathrm{b}}$ is given by

$$
\mathbb{E}\left[\left(\hat{P}_{\mathrm{b}}-P_{\mathrm{b}}\right)^{2}\right]=\frac{1}{N}\left(\mathbb{E}\left[F^{2}(\mathbf{x})\right]-P_{\mathrm{b}}^{2}\right),
$$

which can be reduced by increasing the number of simulation runs $N$. However, when differences in the vectors $\left\{\mathbf{x}_{i}\right\}$ have a great impact on $F\left(\mathbf{x}_{i}\right)$, a sufficient estimation accuracy might require a prohibitively large number of simulation runs $N$, especially when the bit error rate $P_{\mathrm{b}}$ is small.

In order to reduce the required number of simulation runs, IS can be used. In this way, $N^{*}$ vectors $\left\{\mathbf{x}_{i}\right\}$ are generated independently according to a biased distribution $q(\mathbf{x})$, and the BER estimate is computed as

$$
\hat{P}_{\mathrm{b}}^{*} \triangleq \frac{1}{N^{*}} \sum_{i=1}^{N^{*}} F\left(\mathbf{x}_{i}\right) \frac{p\left(\mathbf{x}_{i}\right)}{q\left(\mathbf{x}_{i}\right)},
$$

where the correction factors $p\left(\mathbf{x}_{i}\right) / q\left(\mathbf{x}_{i}\right)$ guarantee an unbiased BER estimate. As the biased distribution $q(\mathbf{x})$ yields an additional degree of freedom, it permits to reduce the variance $\sigma^{* 2}$ of the BER estimate, which is given by

$$
\sigma^{* 2}=\frac{1}{N^{*}}\left(\mathbb{E}^{*}\left[\left(\frac{F(\mathbf{x}) p(\mathbf{x})}{q(\mathbf{x})}\right)^{2}\right]-P_{\mathrm{b}}^{2}\right),
$$

where $\mathbb{E}^{*}[\cdot]$ denotes expectation over the biased distribution $q(\mathbf{x})$. Selecting a proper biased distribution allows to substantially reduce the simulation time to estimate the BER with a given precision as compared to conventional MC simulation, i.e., $N^{*} \ll N$. Obviously, the variance $\sigma^{* 2}$ is minimized when the expectation in (8) is minimized. It is readily verified that $\sigma^{* 2}=0$ when

$$
q(\mathbf{x})=\frac{F(\mathbf{x}) p(\mathbf{x})}{P_{\mathrm{b}}} .
$$

However, the biased distribution resulting from (9) is impractical, as it depends on the unknown bit error rate $P_{\mathrm{b}}$ that is to be estimated by simulation. Nonetheless, (9) indicates that an efficient biased distribution should be proportional to an approximation of $F(\mathbf{x}) p(\mathbf{x})$.

In this contribution, we propose an IS approach where we keep the actual PDFs for the data symbols $\mathbf{s}$ and the additive channel noise $\mathbf{w}$ unchanged, and search for a convenient biased distribution $q(\mathbf{h})$ for the channel $\mathbf{h}$. Hence, we have

$$
\begin{aligned}
& p(\mathbf{x})=p(\mathbf{s}, \mathbf{w}, \mathbf{h})=p(\mathbf{s}, \mathbf{w} \mid \mathbf{h}) p(\mathbf{h}) \\
& q(\mathbf{x})=q(\mathbf{s}, \mathbf{w}, \mathbf{h})=p(\mathbf{s}, \mathbf{w} \mid \mathbf{h}) q(\mathbf{h}) .
\end{aligned}
$$

From (10) and (11), it follows that (8) reduces to

$$
\sigma^{* 2}=\frac{1}{N^{*}}\left(\mathbb{E}^{*}\left[\left(\tilde{F}(\mathbf{h}) \frac{p(\mathbf{h})}{q(\mathbf{h})}\right)^{2}\right]-P_{\mathrm{b}}^{2}\right),
$$

where $\mathbb{E}^{*}[\cdot]$ reduces to averaging over the biased channel distribution $q(\mathbf{h})$ and $\tilde{F}(\mathbf{h})$ is defined as

$$
\tilde{F}(\mathbf{h})=\sqrt{\mathbb{E}_{\mathbf{s}, \mathbf{w} \mid \mathbf{h}}\left[F^{2}(\mathbf{s}, \mathbf{w}, \mathbf{h})\right]},
$$

with $\mathbb{E}_{\mathbf{s}, \mathbf{w} \mid \mathbf{h}}[\cdot]$ denoting expectation over the conditional PDF $p(\mathbf{s}, \mathbf{w} \mid \mathbf{h})$. Taking the similarity of (8) and (12) into account, it follows that $\sigma^{* 2}$ from (12) is minimum for

$$
q(\mathbf{h}) \propto \tilde{F}(\mathbf{h}) p(\mathbf{h})
$$

where $\propto$ denotes proportionality. Since a closed-form expression for $\tilde{F}(\mathbf{h})$ from (13) is usually not available or too complex to yield a practical biased distribution, we rearrange (7) as

$$
\hat{P}_{\mathrm{b}}^{*}=\frac{1}{N_{\mathrm{b}}} \sum_{n=1}^{N_{\mathrm{b}}} \hat{P}_{\mathrm{b}, n}^{*}
$$

where

$$
\hat{P}_{\mathrm{b}, n}^{*}=\frac{1}{N^{*}} \sum_{i=1}^{N^{*}} I_{n}\left(\mathbf{x}_{i}\right) \frac{p\left(\mathbf{x}_{i}\right)}{q\left(\mathbf{x}_{i}\right)}
$$

is the IS estimate of the probability that a detection error for the $n$th bit occurs. In order to find a convenient biased distribution, we look for the distribution $q_{n}(\mathbf{h})$ that minimizes the variance of the individual terms $\hat{P}_{\mathrm{b}, n}^{*}$ rather than the variance of $\hat{P}_{\mathrm{b}}^{*}$. Using the same reasoning that lead to (14), this biased distribution is

$$
q_{n}(\mathbf{h}) \propto \sqrt{P_{\mathrm{b}, n}(\mathbf{h})} p(\mathbf{h}),
$$

where $P_{\mathrm{b}, n}(\mathbf{h})=\mathbb{E}_{\mathbf{s}, \mathbf{w} \mid \mathbf{h}}\left[I_{n}^{2}(\mathbf{s}, \mathbf{w}, \mathbf{h})\right]=\mathbb{E}_{\mathbf{s}, \mathbf{w} \mid \mathbf{h}}\left[I_{n}(\mathbf{s}, \mathbf{w}, \mathbf{h})\right]$ is the error probability of the $n$th bit, conditioned on the channel $\mathbf{h}$. Note that, in general, the obtained biased distribution depends on the considered bit index $n$. The exact expression of the conditional error probability $P_{\mathrm{b}, n}(\mathbf{h})$ depends on the observation model and the type of receiver considered, and is often unknown. Hence, a suitable approximation of $P_{\mathrm{b}, n}(\mathbf{h})$ is usually needed to obtain a biased distribtion $q_{n}(\mathbf{h})$ that adequately reduces the variance of the estimate $\hat{P}_{\mathrm{b}, n}^{*}$. 


\section{ApplicAtion to NAKAgAmi- $m$ FADING CHANNELS}

In this section, we derive a convenient biased channel distribution $q_{n}(\mathbf{h})$ for OSTBCs transmitted on spatially correlated Nakagami- $m$ fading channels. $P_{\mathrm{b}, n}(\mathbf{h})$ in (17) is computed as the conditional bit error probability $P_{\mathrm{b}, n}^{(\mathrm{ML})}(\mathbf{h})$ of a maximumlikelihood (ML) receiver with PCK, which is well approximated by [9]

$$
P_{\mathrm{b}, n}^{(\mathrm{ML})}(\mathbf{h}) \propto Q\left(\sqrt{\beta \frac{E_{\mathrm{s}}}{N_{0}}\|\mathbf{h}\|^{2}}\right),
$$

where $Q(\cdot)$ is the Gaussian $Q$-function, $\beta=2 \lambda$ in case of BPSK, and $\beta=\frac{3 \lambda}{M-1}$ in case of $M$-QAM transmission with Gray mapping. Furthermore, we replace $Q(x)$ in (18) by the Chernoff upper bound $(1 / 2) \exp \left(-x^{2} / 2\right)$ [9], such that the biased distribution resulting from (17) is given by

$$
q_{n}(\mathbf{h}) \propto \exp \left(-\frac{\beta}{4} \frac{E_{s}}{N_{0}}\|\mathbf{h}\|^{2}\right) p(\mathbf{h})
$$

Since (19) is independent of the bit index $n$, the bit index can be dropped and the same biased distribution can be used to reduce the variance of the individual bit error probability estimates $\hat{P}_{\mathrm{b}, n}^{*}$ for $n=1, \cdots, N_{\mathrm{b}}$. Moreover, it is expected to efficiently reduce the variance of the BER estimate $\hat{P}_{\mathrm{b}}^{*}$ as well.

Assuming a MIMO channel vector $\mathbf{h}=\left[h_{1}, \ldots, h_{L}\right]$ containing $L$ complex-valued coefficients $h_{\ell}$, the magnitudes $\alpha_{\ell}=\left|h_{\ell}\right|$ of the channel coefficients are assumed to be distributed according to the Nakagami- $m$ distribution [10]. This versatile statistical distribution is able to accurately model a variety of fading environments by selecting a proper value for the fading parameter $m \geq 1 / 2$ [9] and includes the Rayleigh $(m=1)$ and the one-sided Gaussian $(m=1 / 2)$ distributions as special cases; for $m \rightarrow \infty$, a Nakagami- $m$ fading channel converges to an AWGN channel. The correlation between the channel coefficients is represented by the $L \times L$ power correlation matrix $\boldsymbol{\Sigma}$, the entries of which are defined as [9, Eq. (9.195)]

$$
(\boldsymbol{\Sigma})_{i, n} \triangleq \frac{\operatorname{cov}\left(\alpha_{i}^{2}, \alpha_{n}^{2}\right)}{\sqrt{\operatorname{var}\left(\alpha_{i}^{2}\right) \operatorname{var}\left(\alpha_{n}^{2}\right)}},
$$

where $i, n=1, \ldots, L$. Using the Kronecker model proposed in [11], (20) can be decomposed as

$$
\boldsymbol{\Sigma}=\boldsymbol{\Sigma}_{\mathrm{t}} \otimes \boldsymbol{\Sigma}_{\mathrm{r}}
$$

where $\boldsymbol{\Sigma}_{\mathrm{t}}$ and $\boldsymbol{\Sigma}_{\mathrm{r}}$ are the $L_{\mathrm{t}} \times L_{\mathrm{t}}$ transmit and $L_{\mathrm{r}} \times L_{\mathrm{r}}$ receive power correlation matrices, respectively.

In the case of integer and identical fading parameters, i.e., $m_{\ell}=m, \forall \ell, L$ correlated Nakagami- $m$ RVs $\alpha_{\ell}$ can easily be obtained from $2 m$ i.i.d. random vectors $\mathbf{y}_{k}=$ $\left[y_{k, 1}, y_{k, 2}, \ldots, y_{k, L}\right]^{\mathrm{T}}$, with $k=1, \ldots, 2 m$, as

$$
\alpha_{\ell}^{2} \triangleq \sum_{k=1}^{2 m} y_{k, \ell}^{2}
$$

where $y_{k, \ell}$, with $\ell=1, \ldots, L$, are correlated real-valued zeromean (ZM) Gaussian RVs. According to [12], $\alpha_{\ell}$ 's from (22) are correlated Nakagami- $m$ RVs with $\mathbb{E}\left[\alpha_{\ell}^{2}\right]=\Omega_{\ell}$ and power correlation matrix $\boldsymbol{\Sigma}$, if the covariance matrix $\mathbf{Q}=\mathbb{E}\left[\mathbf{y}_{k} \mathbf{y}_{k}^{\mathrm{T}}\right]$ of the column vectors $\mathbf{y}_{k}$ is given by

$$
\mathbf{Q}=\frac{1}{2 m} \boldsymbol{\Omega}^{\circ \frac{1}{2}} \boldsymbol{\Sigma}_{\mathbf{G}} \boldsymbol{\Omega}^{\circ \frac{1}{2}},
$$

where $\Omega=\operatorname{diag}\left\{\Omega_{1}, \Omega_{2}, \ldots, \Omega_{L}\right\}$ is a $L \times L$ diagonal matrix and $\boldsymbol{\Sigma}_{\mathrm{G}}=\boldsymbol{\Sigma}^{\circ \frac{1}{2}}$, with $\mathbf{X}^{\circ \frac{1}{2}}$ denoting the element-wise square root of a matrix $\mathbf{X}$.

Since the channel coefficients are obtained from auxiliary RVs $y_{k, \ell}$, we derive the biased distribution of the auxiliary RVs rather than of the channel coefficients. By introducing the vector $\mathbf{y}=\left[\mathbf{y}_{1}^{\mathrm{T}}, \mathbf{y}_{2}^{\mathrm{T}}, \ldots, \mathbf{y}_{2 m}^{\mathrm{T}}\right]^{\mathrm{T}}$, we have $\|\mathbf{h}\|^{2}=\|\mathbf{y}\|^{2}=$ $\sum_{k=1}^{2 m} \mathbf{y}_{k}^{\mathrm{T}} \mathbf{y}_{k}$. Moreover, as the vectors $\left\{\mathbf{y}_{k}\right\}$ are i.i.d., the distribution $p(\mathbf{y})$ of the auxiliary $\mathrm{RVs}$ is given by $p(\mathbf{y})=$ $\prod_{k=1}^{2 m} p_{0}\left(\mathbf{y}_{k}\right)$. In this way, it follows from (19) that the biased distribution $q(\mathbf{y})$ reduces to $q(\mathbf{y})=\prod_{k=1}^{2 m} q_{0}\left(\mathbf{y}_{k}\right)$, where

$$
q_{0}\left(\mathbf{y}_{k}\right) \propto \exp \left(-\frac{\beta}{4} \frac{E_{s}}{N_{0}} \mathbf{y}_{k}^{\mathrm{T}} \mathbf{y}_{k}\right) p_{0}\left(\mathbf{y}_{k}\right)
$$

Since the auxiliary RVs $y_{k, \ell}$, with $\ell=1, \ldots, L$, are $\mathrm{ZM}$ Gaussian RVs with covariance matrix $\mathbf{Q}$, the PDF $p_{0}\left(\mathbf{y}_{k}\right)$ of the random vector $\mathbf{y}_{k}$ is given by

$$
p_{0}\left(\mathbf{y}_{k}\right)=\frac{1}{(2 \pi)^{\frac{L}{2}} \sqrt{\operatorname{det}(\mathbf{Q})}} \exp \left(-\frac{1}{2} \mathbf{y}_{k}^{\mathrm{T}} \mathbf{Q}^{-1} \mathbf{y}_{k}\right) .
$$

From (24) and (25), it follows that $q\left(\mathbf{y}_{k}\right)$ is the joint PDF of $L$ correlated ZM Gaussian RVs with a covariance matrix $\mathbf{Q}^{\prime}$ given by

$$
\mathbf{Q}^{\prime}=\left(\mathbf{Q}^{-1}+\frac{\beta}{2} \frac{E_{\mathrm{s}}}{N_{0}} \mathbf{I}_{L}\right)^{-1} .
$$

The distribution $p(\mathbf{y})$ of the auxiliary RVs and the biased distribution $q(\mathbf{y})$ can be easily obtained from (25) and (26), respectively. Taking (10) and (11) into account, the correction factor $p(\mathbf{x}) / q(\mathbf{x})$ in (7) depends on $\mathbf{y}$ only and is given by

$$
\begin{aligned}
\frac{p(\mathbf{x})}{q(\mathbf{x})} & =\frac{p(\mathbf{s}, \mathbf{w}, \mathbf{y})}{q(\mathbf{s}, \mathbf{w}, \mathbf{y})}=\frac{p(\mathbf{y})}{q(\mathbf{y})} \\
& =\frac{\exp \left[-\frac{1}{2} \sum_{k=1}^{2 m} \mathbf{y}_{k}^{\mathrm{T}}\left(\mathbf{Q}^{-1}-\mathbf{Q}^{\prime-\mathbf{1}}\right) \mathbf{y}_{k}\right]}{\left[\operatorname{det}(\mathbf{Q}) \operatorname{det}\left(\mathbf{Q}^{\prime-\mathbf{1}}\right)\right]^{m}} \\
& =\frac{\exp \left(\frac{\beta}{4} \frac{E_{\mathbf{s}}}{N_{0}}\|\mathbf{y}\|^{2}\right)}{\left[\operatorname{det}\left(\mathbf{I}_{L}+\frac{\beta}{2} \frac{E_{\mathrm{s}}}{N_{0}} \mathbf{Q}\right)\right]^{m}} .
\end{aligned}
$$

Although (18) is obtained under the assumption of PCK, it is important to understand that the resulting biased distribution is convenient also in case of imperfect channel estimation (ICE), which we demonstrate in section $\mathrm{V}$, or in the presence of impairments, such as residual frequency offset, IQ imbalance, and phase noise, in which case $P_{\mathrm{b} n}^{(\mathrm{ML})}(\mathbf{h})$ in (18) serves as an approximation of the actual $P_{\mathrm{b}, n}(\mathbf{h})$. The proposed IS technique also applies to SIMO systems with MRC. In addition, it can be extended to the case of multicarrier communication on dispersive MIMO channels, using for instance OSTBCs or orthogonal space-frequency block codes. In multicarrier systems, the relevant channel distribution is the joint distribution of the channel transfer function values at the subcarrier 
frequencies, which is to be derived from the joint distribution of the channel impulse response samples. In the important case of jointly Gaussian channel impulse response samples (i.e., Rayleigh or Rice fading), the channel transfer function values are also jointly Gaussian.

\section{NUMERICAL RESULTS}

In this section, we illustrate the efficiency gain achieved by the biased sampling distribution proposed in section IV. By requiring that the ratio of the variance of the simulated BER to the square of its expectation does not exceed a prescribed value $\epsilon^{2}$, a certain accuracy can be guaranteed for the simulated BER results:

$$
\frac{\operatorname{var}\left[\hat{P}_{\mathrm{b}}\right]}{\left(\mathbb{E}\left[\hat{P}_{\mathrm{b}}\right]\right)^{2}} \leq \epsilon^{2},
$$

where $\hat{P}_{\mathrm{b}}$ is given by (5) or (7). Note that the $N$ realizations $\left\{\mathbf{x}_{i}\right\}$ of the vector $\mathbf{x}$ are independently generated, such that $\operatorname{var}\left[\hat{P}_{\mathrm{b}}\right]=\frac{1}{N} \operatorname{var}[F(\mathbf{x})]$. Hence, for a given accuracy $\epsilon^{2}$, the number of simulation runs $N$ needs to satisfy

$$
N \geq \frac{\operatorname{var}[F(\mathbf{x})]}{\epsilon^{2} P_{\mathrm{b}}^{2}} .
$$

In the following, we compute the lower bound (29) from the simulations by substituting $P_{\mathrm{b}}$ and $\operatorname{var}[F(\mathbf{x})]$ by the sample mean and variance resulting from $\left\{F\left(\mathbf{x}_{i}\right)\right\}$, respectively.

First we consider Alamouti's code [8] transmitted on a $2 \times 2$ Nakagami- $m$ fading channel with fading parameter $m=2$. The channel coefficients, which are assumed to remain constant during one frame, have a power correlation matrix $\boldsymbol{\Sigma}=\boldsymbol{\Sigma}_{\mathrm{t}} \otimes \boldsymbol{\Sigma}_{\mathrm{r}}$, with $\boldsymbol{\Sigma}_{\mathrm{r}}=\mathbf{I}_{2}$ and

$$
\boldsymbol{\Sigma}_{\mathrm{t}}=\left[\begin{array}{cc}
1 & 0.64 \\
0.64 & 1
\end{array}\right]
$$

The receiver performs pilot-based linear minimum meansquare error (LMMSE) channel estimation, followed by mismatched ML detection [13]. To this end, the transmission is organized in frames containing 100 coded data symbols and 14 pilot symbols. Assuming that each vector $\mathrm{x}$ contains exactly one data frame, a fixed number of $N=10^{4}$ data frames is used in the simulations. Fig. 1 displays the BER from MC simulations with and without IS, for 4-QAM and 16-QAM transmission. With IS, accurate BER results down to $10^{-10}$ are obtained using $N=10^{4}$ frames. In particular, for $E_{\mathrm{b}} / N_{0}=20 \mathrm{~dB}$ and 4-QAM, an accuracy of $\epsilon^{2}=0.025$ is obtained. When no IS is used, a substantial deterioration of the accuracy can be observed for BER values below $10^{-4}$; for BER values below $10^{-6}$, the results are totally unreliable. In Fig. 2, we show the number of frames required to obtain an accuracy of $\epsilon^{2}=0.025$ with and without using IS. It can be observed that substantial run-time savings can be achieved using the proposed IS technique. In addition, 16QAM requires fewer frames than 4-QAM to achieve a given estimation accuracy, since the former constellation gives rise to a larger BER than 4-QAM.

We can specify the run-time savings attained by IS by defining the efficiency gain $\gamma_{\mathrm{IS}}$ as the ratio of the number of frames required to achieve a certain accuracy $\epsilon^{2}$ by using

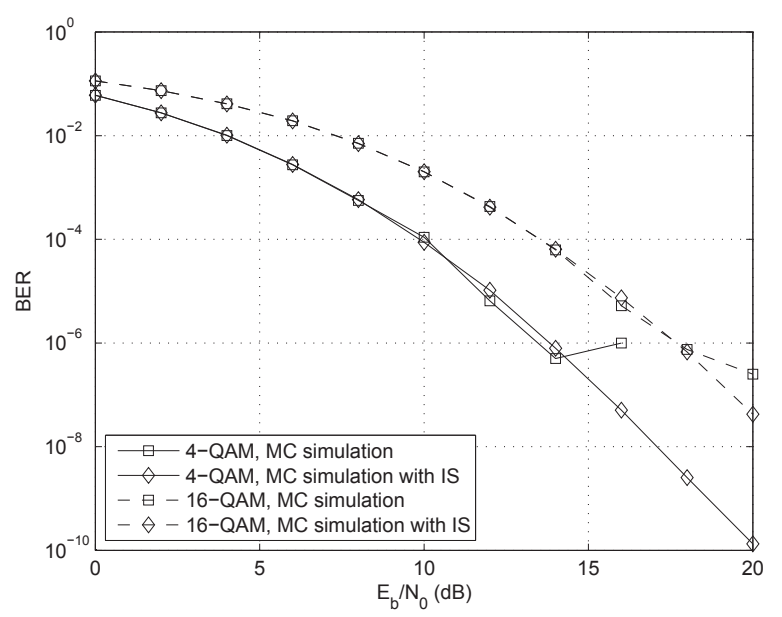

Fig. 1. BER for Alamouti's code on $2 \times 2$ Nakagami channel with ICE.

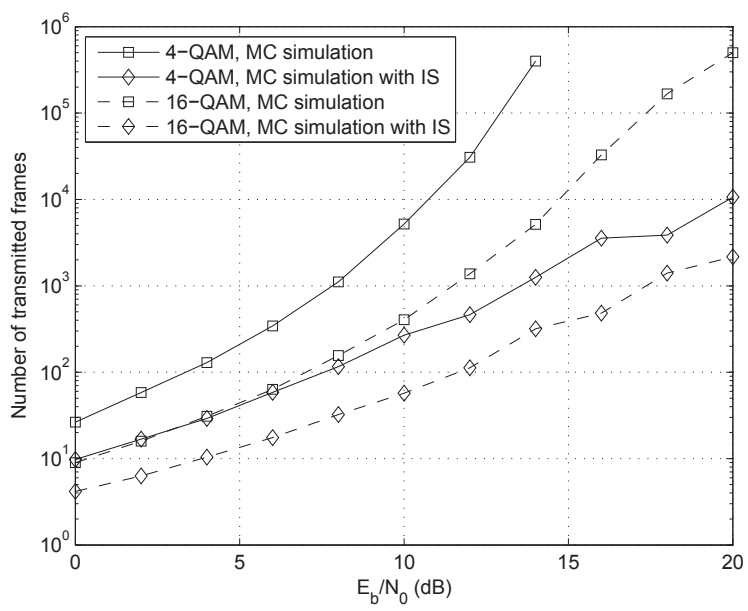

Fig. 2. Number of transmitted frames required to ensure $\epsilon^{2}=0.025$ using Alamouti's code on $2 \times 2$ Nakagami channel with ICE.

straightforward MC simulations to the number of frames required to achieve the same accuracy when using IS. It follows from (29) that

$$
\gamma_{\mathrm{IS}}=\frac{\left.\operatorname{var}[F(\mathbf{x})]\right|_{\mathrm{MC}}}{\left.\operatorname{var}[F(\mathbf{x})]\right|_{\mathrm{IS}}},
$$

where the variances $\left.\operatorname{var}[F(\mathbf{x})]\right|_{\text {IS }}$ and $\left.\operatorname{var}[F(\mathbf{x})]\right|_{\text {MC }}$ for MC simulations with and without IS, respectively, are obtained from the MC simulation results $\left\{F\left(\mathbf{x}_{i}\right)\right\}$ conducted with and without using IS. In order to investigate the impact of spatial correlation and the number of receive antennas on the efficiency gain, we consider Alamouti's code transmitted on a Nakagami- $m$ fading channel with $m=2$ and captured by an ML receiver equipped with 1,2 , or 3 antennas. The power correlation matrix of the MIMO channel is given by $\boldsymbol{\Sigma}=\boldsymbol{\Sigma}_{\mathrm{t}} \otimes \boldsymbol{\Sigma}_{\mathrm{r}}$, where the diagonal elements of $\boldsymbol{\Sigma}_{\mathrm{t}}$ and $\boldsymbol{\Sigma}_{\mathrm{r}}$ are assumed to be given by 1 and the non-diagonal elements are given by $\rho$. Furthermore, the data frames consist of 100 coded data symbols belonging to a 4-QAM constellation and 14 pilot symbols per transmit antenna used for LMMSE channel esti- 


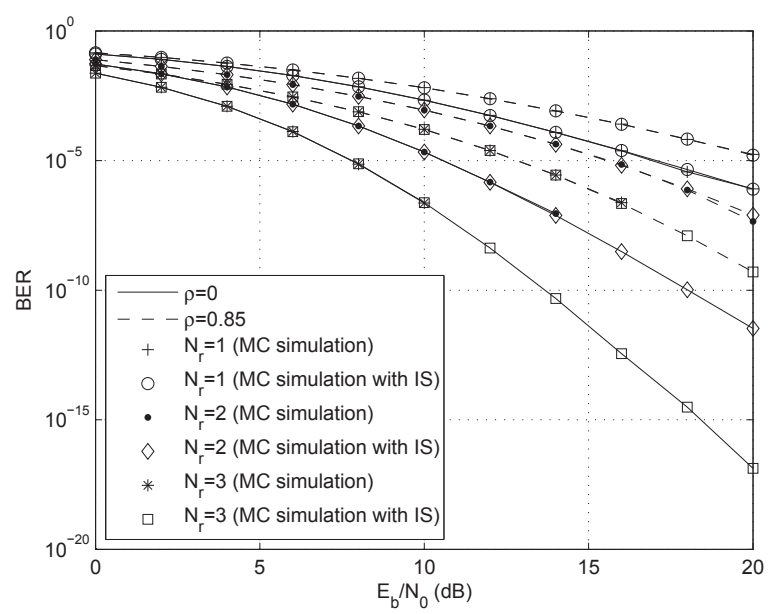

Fig. 3. BER for Alamouti's code on Nakagami channel with ICE.

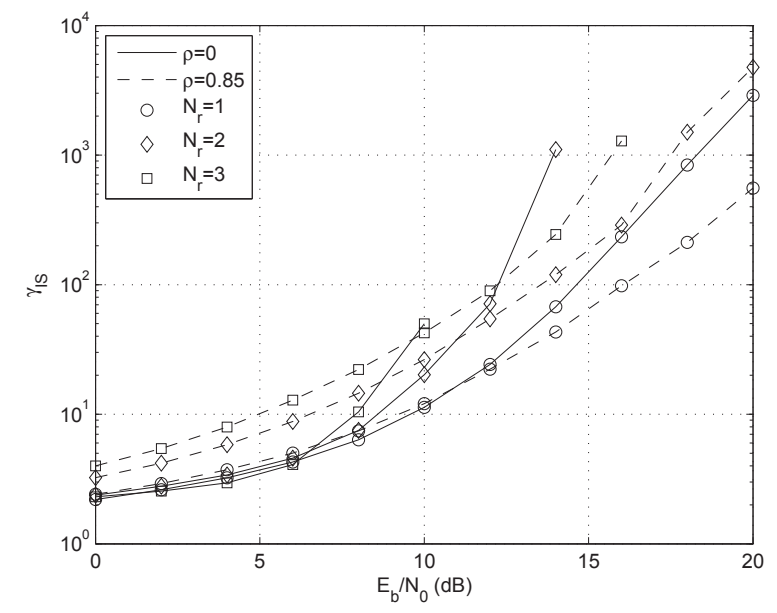

Fig. 4. Efficiency gain $\gamma_{\text {IS }}$ attained by IS for Alamouti's code on Nakagami channel with ICE.

mation. Fig. 3 displays the simulated BER for a fixed number of $N=10^{6}$ data frames transmitted. Whereas employing IS enables accurate BER results down to $10^{-17}$, straightforward MC simulation manages to obtain reliable BER values down to about $10^{-7}$ only. In addition, spatial correlation with $\rho=0.85$ results in a substantial BER degradation as compared to the uncorrelated MIMO channel $(\rho=0)$. In Fig. 4 , the efficiency gain $\gamma_{\mathrm{IS}}$ is shown for the BER curves from Fig. 3. For SNR values below $10 \mathrm{~dB}$, efficiency gains between 2 and 50 are achieved. For high SNR, however, rapidly increasing efficiency gains up to $5 * 10^{3}$ can be appreciated. Even higher efficiency gains are to be expected for the receiver with 3 antennas and, in case of uncorrelated channels, the dual-antenna receiver. In general, it is observed for high SNR that the efficiency gain grows when the BER decreases, i.e., for decreasing level of spatial correlation and increasing number of receive antennas. Hence, even when very low BERs are targeted, the proposed IS sampling distribution enables practical BER simulation for OSTBC systems on spatially correlated Nakagami channels.

\section{CONCLUSIONS}

In this contribution, we presented a simple IS technique that allows to increase by orders of magnitude the efficiency of BER simulations for OSTBC systems on spatially correlated Nakagami- $m$ fading channels. While keeping the distributions for the channel noise and the data symbols unchanged, we proposed a convenient biased distribution for the fading channel.

\section{ACKNOWLEDGEMENTS}

Part of this research has been funded by the Interuniversity Attraction Poles Programme initiated by the Belgian Science Policy Office.

\section{REFERENCES}

[1] P. Glynn and D. Iglehart, "Importance sampling for stochastic simulation," Management Sci., vol. 35, pp. 1367-1392, 1989.

[2] K. Shanmugam and P. Balaban, "A modified monte-carlo simulation technique for the evaluation of error rate in digital communication systems," IEEE Trans. Commun., vol. 28, no. 11, pp. 1916 - 1924, Nov. 1980.

[3] D. Lu and K. Yao, "Improved importance sampling technique for efficient simulation of digital communication systems," IEEE J. Select. Areas Commun., vol. 6, no. 1, pp. 67 -75, Jan. 1988.

[4] D. I. Kim and D. I. Lee, "CQ importance sampling technique for efficient simulation in Rayleigh-faded channels," in Proc. IEEE Int. Symp. on Information Theory (ISIT), Aug. 1998, p. 158

[5] W. Al-Qaq and J. Townsend, "A stochastic importance sampling methodology for the efficient simulation of adaptive systems in frequency nonselective Rayleigh fading channels," IEEE Journal on Selected Areas in Communications, vol. 15, no. 4, pp. 614 -625, May 1997.

[6] K. C. Nguyen, U. Gunawardana, and R. Liyana-Pathirana, "Application of importance sampling for simulation of orthogonal space-time block coded systems," in Asia-Pacific Conference on Communications (APCC), Oct 2005, pp. 986-990.

[7] L. Jacobs, G. Alexandropoulos, M. Moeneclaey, H. Bruneel, and P. Mathiopoulos, "Analysis and efficient evaluation of the BER of OSTBCs with imperfect channel estimation in arbitrarily correlated fading channels," IEEE Trans. Signal Process., vol. 59, no. 6, pp. 27202733, Jun. 2011.

[8] V. Tarokh, H. Jafarkhani, and A. R. Calderbank, "Space-time block codes from orthogonal designs," IEEE Trans. Inform. Theory, vol. 45 , no. 5, pp. 1456-1467, Jul. 1999.

[9] M. K. Simon and M.-S. Alouini, Digital Communication over Fading Channels, 2nd ed. New York: Wiley, 2005.

[10] M. Nakagami, "The $m$-distribution-A general formula for intensity distribution of rapid fading," in Statistical Methods in Radio Wave Propagation. New York: Pergamon, 1960, pp. 3-36.

[11] K. I. Pedersen, J. B. Andersen, J. P. Kermoal, and P. Mogensen, "A stochastic multiple-input-multiple-output radio channel model for evaluation of space-time coding algorithms," in Proc. IEEE Vehicular Technology Conference (VTC-Fall), vol. 2, Boston, MA, USA, 24-28 Sep. 2000, pp. 893-897.

[12] G. C. Alexandropoulos, N. C. Sagias, F. I. Lazarakis, and K. Berberidis, "New results for the multivariate Nakagami- $m$ fading model with arbitrary correlation matrix and applications," IEEE Trans. Wireless Commun., vol. 8, no. 1, pp. 245-255, Jan. 2009.

[13] L. Jacobs and M. Moeneclaey, "BER analysis of square OSTBCs with LMMSE channel estimation in arbitrarily correlated Rayleigh fading channels," IEEE Commun. Lett., vol. 14, no. 7, pp. 626-628, Jul. 2010 